\title{
Fatores associados à obesidade geral e ao percentual de gordura corporal em mulheres no climatério da cidade de São Paulo, Brasil
}

\author{
Factors associated with general obesity and the percentage of body \\ fat of women during the menopause in the city of São Paulo, Brazil
}

Ana Paula França ${ }^{1}$

Maria de Fátima Nunes Marucci ${ }^{1}$

Maria de Lourdes do Nascimento da Silva ${ }^{1}$

Manuela de Almeida Roediger ${ }^{1}$

${ }^{1}$ Faculdade de Saúde Pública, Universidade de São Paulo. Av. Dr. Arnaldo 715, Cerqueira César. 01246-904 São Paulo SP Brasil.apfranca@usp.br

\begin{abstract}
To verify the association between obesity and demographic, clinical and lifestyle variables in climacteric women, a cross-sectional study was conducted in outpatient clinics, with 469 women aged 40 to 65 years in the city of São Paulo, Brazil. The dependent variables were: obesity according to body mass index (BMI) and obesity according to percentage of body fat (\% BF). The main explanatory variable was: climacteric phase (pre or postmenopausal); and control variables were: age; years of formal study; parity; menopausal hormone therapy (MHT) use; physical activity practice and smoking habit. Multiple regression analysis was performed using the Stata 9.2 software. According to the BMI, obesity was positively associated with parity $(R R=1.62,95 \% C I=1.11$ 2.37) and, negatively, with years of formal study $(R P=0.71, C I 95 \%=0.55-0.91)$ and with physical activity practice $(P R=0.45,95 \% C I=0.33$ 0.61 ). According to the $\% \mathrm{BF}$, obesity was positively associated with parity $(P R=1.60,95 \% C I=$ 1.03-2.49) and negatively with physical activity practice $(P R=0.43 ; 95 \% C I=0.29-0.63)$. While being active physically was protective, multiparity was a risk factor for developing obesity for women in this study.
\end{abstract}

Key words Obesity, Parity, Physical activity, Climacteric, Menopause
Resumo Para verificar a associação entre obesidade e variáveis demográficas, clinicas e relacionadas ao estilo de vida em mulheres no climatério, foi realizado um estudo transversal com 469 mulheres de 40 a 65 anos em dois ambulatórios públicos da cidade de São Paulo. As variáveis dependentes foram: obesidade, segundo indice de massa corporal (IMC) e obesidade, segundo percentual de gordura corporal (\%GC). A variável explanatória principal foi: fase do climatério (pré ou pós-menopausa); e as variáveis de controle foram: idade; anos de estudo; paridade; uso de terapia hormonal da menopausa; prática de atividade física e hábito de fumar. Realizou-se análise de regressão "glm" múltipla, utilizando para as análises o software Stata 9.2. Segundo o IMC, a obesidade associou-se positivamente, à paridade ( $R P=$ 1,62; IC 95\% = 1,11-2,37) e, negativamente, aos anos de estudo $(R P=0,71 ;$ IC 95\% = 0,55-0,91) e à prática de atividade física $(R P=0,45 ; I C 95 \%$ =0,33-0,61). De acordo com o \%GC, a obesidade associou-se positivamente à paridade $(R P=1,60$; IC 95\% = 1,03-2,49) e, negativamente, à prática de atividade física ( $R P=0,43$; IC 95\% = 0,290,63). Enquanto a prática de atividade física foi um fator protetor, a multiparidade constituiu-se como fator de risco para a prevalência de obesidade no grupo de mulheres deste estudo.

Palavras-chave Obesidade, Paridade, Atividade física, Climatério, Menopausa 


\section{Introdução}

Embora existam diferenças nas prevalências e tendências de acordo com o grupo etário e estrato social, praticamente todos os países estão vivenciando uma epidemia de obesidade, impulsionada, em grande parte, pela liberalização do comércio global, o crescimento econômico e a rápida urbanização $0^{1,2}$.

Em todo o mundo, o índice de massa corporal (IMC) aumentou em média, por década, 0,4 $\mathrm{kg} / \mathrm{m} 2$ entre os homens e $0,5 \mathrm{~kg} / \mathrm{m} 2$ entre as mulheres, entre 1980 e $2008^{3}$. Em 2014, mais de 1,9 bilhões de adultos apresentavam excesso de peso (IMC $\geq 25 \mathrm{~kg} / \mathrm{m} 2$ ) e mais de 600 milhões eram obesos $(\mathrm{IMC} \geq 30 \mathrm{~kg} / \mathrm{m} 2)^{4}$.

O risco de doenças cardíacas, acidentes vasculares encefálicos, diabetes e certos tipos de câncer aumentam progressivamente com o aumento do IMC ${ }^{5,6}$. Das 57 milhões de mortes ocorridas no mundo em 2008, 36 milhões - quase dois terços foram atribuídas a doenças não transmissíveis ${ }^{6,7}$.

Em 2011, no Brasil, as doenças crônicas não transmissíveis foram responsáveis por $72,7 \%$ do total de mortes, com destaque para as doenças do aparelho circulatório (30,4\% dos óbitos), as neoplasias $(16,4 \%)$ e o diabetes $(5,3 \%)^{8}$. Embora, neste país, a taxa de mortalidade geral por doenças não transmissíveis tenha apresentado queda de $20 \%$ entre 1996 e 2007, principalmente ao sucesso da implementação de políticas de saúde voltadas à diminuição do tabagismo e à expansão da atenção primária, a prevalência de diabetes e hipertensão aumentaram, em paralelo ao aumento do excesso de peso, associados às mudanças desfavoráveis na qualidade da dieta e no padrão de atividade física ${ }^{9}$.

A prevalência de excesso de peso ainda é maior nos países de renda média alta, contudo, elevação mais rápida está ocorrendo nos países de renda média baixa ${ }^{4}$. Em algumas regiões do mundo, como nas Américas, mais de 50\% das mulheres estão acima do peso ${ }^{7}$. Nas capitais brasileiras e no Distrito Federal, no período de 2006 a 2012, observou-se aumento na prevalência de obesidade de $11,4 \%$ para $17,4 \%$ em adultos. Neste período, o aumento da prevalência nos homens foi semelhante entre todos grupos socioeconômicos, enquanto no grupo de mulheres a prevalência de obesidade aumentou naquelas com 8 anos ou menos de escolaridade ${ }^{9,10}$.

É fato que a obesidade se desenvolve somente quando, cronicamente, a ingestão excede o gasto de energia, contudo, inúmeros estudos têm mostrado que fatores sociodemográficos e compor- tamentais estão associados ao aumento do peso corporal $^{11-14}$.

Apesar do elevado e crescente índice de obesidade entre as mulheres brasileiras, poucas investigações foram feitas sobre os fatores que poderiam estar associados à esta afecção, sobretudo naquelas que estão na fase do climatério. Nesse contexto, o objetivo deste trabalho é identificar a prevalência de obesidade e sua associação com variáveis sociodemográficas, clínicas e relacionadas ao estilo de vida em mulheres de 40 a 65 anos atendidas em dois ambulatórios públicos da cidade de São Paulo, SP.

\section{Material e métodos}

\section{Delineamento e amostra estudada}

Estudo transversal realizado no Ambulatório de Saúde da Mulher no Climatério (ASMUC), do Centro de Saúde Escola "Geraldo de Paula Souza" da Faculdade de Saúde Pública da USP, no período de Abril a Setembro de 2002, e no Ambulatório de Ginecologia do Hospital Universitário da Universidade de São Paulo (HU/USP), no período de janeiro de 2006 a fevereiro de 2007.

Nos períodos citados, todas as mulheres inscritas nos referidos serviços foram convidadas a participar do estudo, considerando como critérios de inclusão ter idade entre 40 e 65 anos completos. Os critérios de exclusão incluíram: ter diagnóstico de doença cardiovascular, ter sido submetida à histerectomia ou ooforectomia parcial, e o não cumprimento do protocolo para a realização do exame de bioimpedância elétrica (realização de atividade física intensa no dia anterior, sauna nas últimas 8 horas e/ou ingestão de bebidas alcoólicas nas últimas 12 horas). A pesquisa foi aprovada pelos Comitês de Ética em Pesquisa da Faculdade de Saúde Pública e do Hospital Universitário da USP, e as mulheres que aceitaram participar do estudo assinaram o termo de consentimento livre e esclarecido.

\section{Variáveis de estudo}

A variável dependente foi obesidade, identificada pelo índice de massa corporal (IMC = peso/ estatura $\left.{ }^{2}\right)$, adotando-se a classificação da Organização Mundial da Saúde ${ }^{15}$ : não obesa $(<30,0$ $\left.\mathrm{kg} / \mathrm{m}^{2}\right)$ ou obesa $\left(\geq 30,0 \mathrm{~kg} / \mathrm{m}^{2}\right)$; e de acordo com o percentual de gordura corporal (\%GC), obtido pelo método de bioimpedância elétrica (BIA), utilizando-se aparelho portátil RJL-SYSTEMS e 
adotada a classificação sugerida por Gallagher et al. ${ }^{11}$ : não obesa $(<40 \%$ em mulheres de 40 a 59 anos e $<42 \%$ em mulheres de 60 a 65 anos) ou obesa ( $\geq 40 \%$ em mulheres de 40 a 59 anos e $\geq$ $42 \%$ em mulheres de 60 a 65 anos).

A variável explanatória principal foi fase do climatério, considerando pré-menopausa (ciclo menstrual regular e amenorreia por até 11 meses) ou pós-menopausa (amenorreia por período igual ou superior a 12 meses consecutivos e ooforectomia bilateral).

As variáveis de controle foram: idade (em anos completos); anos de estudo ( $<7$ anos ou $\geq 8$ anos); nível de atividade física (sedentária e insuficientemente ativa ou ativa e muito ativa); paridade (0, 1 e 2 partos ou 3 e mais partos); uso de terapia hormonal da menopausa (não usuária e usuária há menos de 12 meses ou usuária por pelo menos 12 meses); hábito de fumar (nunca fumaram e ex-tabagistas ou tabagistas).

O nível de atividade física foi identificado pelo Questionário Internacional de Atividade Física - International Physical Activity Questionnaire-IPAQ, desenvolvido pela $\mathrm{OMS}^{16}$ e validado para a população brasileira adulta de 20 a 34 anos $^{16}$ e em mulheres idosas ${ }^{17}$.

\section{Análise estatística}

Para verificar a associação entre cada variável dependente (obesidade segundo o IMC e segundo o \%GC), a explanatória principal (fase do climatério) e as variáveis de controle (idade, anos de estudo, paridade, nível de atividade física, hábito de fumar e uso de terapia hormonal da menopausa), foram realizados os testes $t$ de student e o qui quadrado de Pearson, anotando-se o valor p descritivo do teste.

Para cada variável dependente foi feita modelagem mediante regressão glm (general linear model) múltipla, com família binomial e ligação ("link") logarítmica, permitindo a obtenção direta de razões de prevalências (RP). Na primeira etapa da modelagem, além da variável explanatória principal, foram incluídas as variáveis de controle com $\mathrm{p}<0,20$ nas análises bivariadas. Eventuais interações foram examinadas, entre a variável explanatória principal (fase do climatério) e as demais variáveis, para teste de interação no modelo múltiplo. Em etapas subsequentes, as variáveis de controle com $\mathrm{p}>0,10$ e as variáveis de interação com $\mathrm{p}>0,05$ foram excluídas do modelo. Todas as análises foram realizadas utilizando o software Stata 9.2.

\section{Resultados}

Foram entrevistadas 469 mulheres de 40 a 65 anos, sendo 172 pacientes ASMUC (FSP/USP) e 297 do Ambulatório de Ginecologia do Hospital Universitário da Universidade de São Paulo (HU/ USP).

Estavam no período de pré-menopausa $49,9 \%$ das mulheres $(41,2 \%$ menstruavam regularmente e $8,7 \%$ não menstruavam há menos de 12 meses); enquanto 50,1\% não menstruavam há mais de 12 meses, caracterizando a pós-menopausa. A maior parte das mulheres tinha entre 45 a 54 anos, sendo a média etária de 51,3 anos (mediana: 51,0 anos; desvio-padrão: 6,9 anos).

A maioria $(55,4 \%)$ referiu ter pelo menos oito anos de estudo; 47,3\% referiram ter três ou mais partos, sendo, em média, 2,5 partos/mulher. Quanto ao nível de atividade física, 55,4\% eram sedentárias ou insuficientemente ativas. Sobre o hábito de fumar atual, 85,9\% das mulheres relataram não fumar e, dentre estas, $23,2 \%$ eram ex-tabagistas. Do total das mulheres, 13,2\% utilizavam terapia hormonal da menopausa oral e referiram utilizar, em média, por 3,0 anos.

A prevalência de obesidade, segundo o IMC, foi 32,0\%. Na análise bivariada o nível de atividade física, paridade, anos de estudo e idade associaram-se à obesidade segundo o IMC $(\mathrm{p}<0,05)$ (Tabela 1).

No modelo múltiplo, a paridade $(\mathrm{RP}=1,62$; $\mathrm{p}=0,012$ ) associou-se positivamente à obesidade segundo o IMC, enquanto o nível de atividade física $(R P=0,45 ; p=0,000)$ e ter oito ou mais anos de estudo $(\mathrm{RP}=0,71 ; \mathrm{p}=0,008)$ associaram-se negativamente a este desfecho. Portanto, no presente estudo, ser sedentária ou insuficientemente ativa, ter três ou mais partos e até sete anos de estudo implicou maior risco de obesidade. É importante ressaltar que a fase do climatério, apesar de não se manter estatisticamente significativa $(p=0,071)$, permaneceu no modelo final por ter sido definida como explanatória principal (Tabela 2).

A prevalência de obesidade, segundo o \%GC, foi $24,7 \%$. O nível de atividade física e a paridade associaram-se à obesidade segundo o \%GC ( $\mathrm{p}<$ 0,05), na análise bivariada (Tabela 3 ).

No modelo múltiplo, a paridade $(\mathrm{RP}=1,60$; $\mathrm{p}=0,035)$ manteve-se positivamente associada à obesidade, segundo o \%GC, enquanto o nível de atividade física permaneceu negativamente associado $(R P=0,43 ; p=0,000)$ ao mesmo desfecho, ou seja, ter três ou mais partos e ser sedentária ou insuficientemente ativa implicou maior risco 
Tabela 1. Distribuição das mulheres de 40 a 65 anos atendidas em ambulatórios públicos da cidade de São Paulo, segundo obesidade (identificada pelo IMC) e variáveis sociodemograficas, clínicas e relacionadas ao estilo de vida.

\begin{tabular}{|c|c|c|c|c|c|c|}
\hline \multirow{3}{*}{ Variáveis explanatórias } & \multicolumn{4}{|c|}{ Obesidade (IMC) } & \multirow{3}{*}{$\mathbf{R P}$} & \multirow{3}{*}{$\begin{array}{c}\text { valor } \\
\text { de } p\end{array}$} \\
\hline & \multicolumn{2}{|c|}{$\begin{array}{c}\text { Não } \\
(\mathbf{n}=319)\end{array}$} & \multicolumn{2}{|c|}{$\begin{array}{c}\text { Sim } \\
(n=150)\end{array}$} & & \\
\hline & $(\mathbf{n})$ & $(\%)$ & $(\mathbf{n})$ & $(\%)$ & & \\
\hline \multicolumn{7}{|l|}{ Fase do climatério } \\
\hline Pré-menopausa $(\mathrm{n}=234)$ & 167 & 71,4 & 67 & 28,6 & 1 & $0,122^{\star}$ \\
\hline Pós-menopausa (n= 235) & 152 & 64,7 & 83 & 35,3 & 1,23 & \\
\hline \multicolumn{7}{|l|}{ Terapia hormonal da menopausa } \\
\hline Nunca usou, já usou ou usa há menos de 12 meses $(\mathrm{n}=415)$ & 277 & 66,7 & 138 & 33,3 & 1 & $0,127^{\star *}$ \\
\hline Usa por, no mínimo, 12 meses $(\mathrm{n}=54)$ & 42 & 77,8 & 12 & 22,2 & 0,67 & \\
\hline \multicolumn{7}{|l|}{ Hábito de fumar } \\
\hline Nunca fumou ou ex-tabagista $(\mathrm{n}=404)$ & 273 & 67,6 & 131 & 32,4 & 1 & 0,614 \\
\hline Tabagista $(\mathrm{n}=65)$ & 46 & 70,8 & 19 & 29,2 & 0,90 & \\
\hline \multicolumn{7}{|l|}{ Nível de atividade física } \\
\hline Sedentária ou insuficientemente ativa $(\mathrm{n}=260)$ & 149 & 57,3 & 111 & 42,7 & 1 & $0,000^{* *}$ \\
\hline Ativa ou muito ativa $(\mathrm{n}=209)$ & 170 & 81,3 & 39 & 18,7 & 0,44 & \\
\hline \multicolumn{7}{|l|}{ Paridade } \\
\hline 0,1 ou 2 partos $(\mathrm{n}=120)$ & 97 & 80,8 & 23 & 19,2 & 1 & $0,001^{\star *}$ \\
\hline 3 ou mais partos $(\mathrm{n}=349)$ & 222 & 63,6 & 127 & 36,4 & 1,90 & \\
\hline \multicolumn{7}{|l|}{ Anos de estudo } \\
\hline Até 7 anos $(\mathrm{n}=209)$ & 128 & 61,2 & 81 & 38,8 & 1 & $0,005^{\star *}$ \\
\hline 8 anos ou mais $(n=260)$ & 191 & 73,5 & 69 & 26,5 & 0,68 & \\
\hline \multicolumn{7}{|l|}{ Idade (variável contínua) } \\
\hline Média & \multicolumn{2}{|c|}{50,9} & \multicolumn{2}{|c|}{52,3} & & $0,043^{\star *}$ \\
\hline
\end{tabular}

de obesidade. Mais uma vez, ressalta-se que, apesar da variável fase do climatério não se manter estatisticamente significativa ( $\mathrm{p}=0,373)$, permaneceu no modelo final por ter sido definida como explanatória principal (Tabela 4).

\section{Discussão}

Neste estudo, mais de um terço das mulheres apresentaram obesidade, identificada pelo IMC. As tendências de elevação na prevalência de obesidade na população feminina em países em desenvolvimento, como o Brasil, mais do que triplicou nos últimos 40 anos, de acordo com inquéritos populacionais nacionais: era de 5,3\% em $1975^{18}, 10,2 \%$ em $1996^{18}, 13,1 \%$ em $2003^{20}$ e $16,9 \%$ em $2009^{21}$. Inquéritos telefônicos para a vigilância de fatores de risco e proteção para doenças crônicas (VIGITEL), realizados na população brasileira, também mostraram aumento da prevalência de obesidade em mulheres com mais de 18 anos na última década: era de $11,5 \%$ em $2006^{9}, 17,3 \%$ em $2012^{10}$ e $17,9 \%$ em $2014^{21}$.

Estudos populacionais também mostram que a prevalência de obesidade é ainda maior nas mulheres acima dos 40 anos. Nos Estados Unidos, $39,5 \%$ das mulheres de 40 a 59 anos eram obesas em 2011-2012 22. No Brasil, dados da Pesquisa de Orçamentos Familiares de 2008-2009 mostraram que $21,5 \%$ e $26,0 \%$ das mulheres brasileiras de 45-54 e 55-64 anos, respectivamente, estavam obesas $^{20}$. Resultados do VIGITEL também mostraram maior prevalência de obesidade em mulheres desses mesmos grupos etários, comparando-se às mulheres adultas, além de aumento na prevalência no período de 2006 a 2012: de 15,1\% para 24,3\% nas mulheres de $45-54$ anos, e de $20,4 \%$ para $25,7 \%$ nas mulheres de $55-64$ anos $^{9,10}$. Comparando-se aos resultados mencionados, a prevalência de obesidade constatada no presente estudo foi substancialmente maior, o que poderia ser atribuído não apenas a diferenças regionais, como também por se tratar de amostra cadas- 
Tabela 2. Modelo da regressão " $g l m$ " (general linear model), da obesidade (identificada pelo IMC), em mulheres de 40 a 65 anos, atendidas em ambulatórios públicos da cidade de São Paulo.

\begin{tabular}{|c|c|c|c|c|c|c|c|c|c|c|}
\hline \multirow[b]{2}{*}{$\begin{array}{c}\text { Variáveis } \\
\text { Explanatórias }\end{array}$} & \multicolumn{2}{|c|}{$1^{\text {a }}$ etapa } & \multicolumn{2}{|c|}{$2^{\mathrm{a}}$ etapa } & \multicolumn{2}{|c|}{$3^{a}$ etapa } & \multicolumn{2}{|c|}{$4^{\mathrm{a}}$ etapa } & \multicolumn{2}{|c|}{ Modelo Final } \\
\hline & $\begin{array}{c}\text { RP } \\
\text { (IC 95\%) }\end{array}$ & $\begin{array}{l}\text { valor } \\
\text { de p }\end{array}$ & $\begin{array}{c}\text { RP } \\
\text { (IC 95\%) }\end{array}$ & $\begin{array}{l}\text { valor } \\
\text { de p }\end{array}$ & $\begin{array}{c}\text { RP } \\
\text { (IC 95\%) }\end{array}$ & $\begin{array}{l}\text { valor } \\
\text { de p }\end{array}$ & $\begin{array}{c}\text { RP } \\
(\text { IC 95\%) }\end{array}$ & $\begin{array}{l}\text { valor } \\
\text { de p }\end{array}$ & $\begin{array}{c}\text { RP } \\
(\text { IC 95\%) }\end{array}$ & $\begin{array}{l}\text { valor } \\
\text { de p }\end{array}$ \\
\hline \multicolumn{11}{|l|}{ Fase do climatério } \\
\hline Pré-menopausa & 1 & & 1 & & 1 & & 1 & & 1 & \\
\hline Pós-menopausa & $\begin{array}{c}1,42 \\
(0,98-2,06)\end{array}$ & 0,068 & $\begin{array}{c}1,33 \\
(1,03-1,71)\end{array}$ & 0,027 & $\begin{array}{c}1,25 \\
(0,90-1,62)\end{array}$ & 0,071 & $\begin{array}{c}1,43 \\
(1,02-2,02)\end{array}$ & 0,041 & $\begin{array}{c}1,25 \\
(0,90-1,62)\end{array}$ & 0,071 \\
\hline \multicolumn{11}{|l|}{$\begin{array}{l}\text { Terapia hormonal da } \\
\text { menopausa }\end{array}$} \\
\hline $\begin{array}{l}\text { Nunca usou, já usou } \\
\text { ou usa há menos de } 12 \\
\text { meses }\end{array}$ & 1 & & 1 & & & & & & & \\
\hline $\begin{array}{l}\text { Usa por no mínimo } 12 \\
\text { meses }\end{array}$ & $\begin{array}{c}0,66 \\
(0,41-1,08)\end{array}$ & 0,098 & $\begin{array}{c}0,66 \\
(0,41-1,09)\end{array}$ & 0,104 & & & & & & \\
\hline \multicolumn{11}{|l|}{ Nível de atividade física } \\
\hline $\begin{array}{l}\text { Sedentária ou } \\
\text { insuficientemente ativa }\end{array}$ & 1 & & 1 & & 1 & & 1 & & 1 & \\
\hline Ativa ou muito ativa & $\begin{array}{c}0,45 \\
(0,33-0,62)\end{array}$ & 0,000 & $\begin{array}{c}0,46 \\
(0,33-0,62)\end{array}$ & 0,000 & $\begin{array}{c}0,45 \\
(0,33-0,61)\end{array}$ & 0,000 & $\begin{array}{c}0,45 \\
(0,33-0,62)\end{array}$ & 0,000 & $\begin{array}{c}0,45 \\
(0,33-0,61)\end{array}$ & 0,000 \\
\hline \multicolumn{11}{|l|}{ Paridade } \\
\hline 0,1 ou 2 partos & 1 & & 1 & & 1 & & 1 & & 1 & \\
\hline 3 ou mais partos & $\begin{array}{c}1,59 \\
(1,09-2,33)\end{array}$ & 0,016 & $\begin{array}{c}1,57 \\
(1,08-2,30)\end{array}$ & 0,017 & $\begin{array}{c}1,62 \\
(1,11-2,37)\end{array}$ & 0,012 & $\begin{array}{c}1,63 \\
(1,12-2,34)\end{array}$ & 0,011 & $\begin{array}{c}1,62 \\
(1,11-2,37)\end{array}$ & 0,012 \\
\hline \multicolumn{11}{|l|}{ Anos de estudo } \\
\hline Até 7 anos & 1 & & 1 & & 1 & & 1 & & 1 & \\
\hline 8 anos ou mais & $\begin{array}{c}0,70 \\
(0,55-0,90)\end{array}$ & 0,006 & $\begin{array}{c}0,71 \\
(0,55-0,91)\end{array}$ & 0,007 & $\begin{array}{c}0,71 \\
(0,55-0,91)\end{array}$ & 0,008 & $\begin{array}{c}0,85 \\
(0,57-1,26)\end{array}$ & 0,418 & $\begin{array}{c}0,71 \\
(0,55-0,91)\end{array}$ & 0,008 \\
\hline \multicolumn{11}{|l|}{ Idade } \\
\hline (variável contínua) & $\begin{array}{c}0,99 \\
(0,97-1,02)\end{array}$ & 0,687 & & & & & & & & \\
\hline \multicolumn{11}{|l|}{$\begin{array}{l}\text { Fase do climatério x grau } \\
\text { de instrução }\end{array}$} \\
\hline (variável de interação) & & & & & & & $\begin{array}{c}0,73 \\
(0,44-1,23)\end{array}$ & 0,239 & & \\
\hline
\end{tabular}

$\mathrm{RP}$ - razão de prevalências.

trada em ambulatórios específicos de atenção às mulheres na fase do climatério.

Além da utilização do IMC para diagnóstico e classificação da obesidade, desde o início da década passada, observa-se aumento do interesse em medidas que discriminem a quantidade de gordura corporal e sua relação às doenças relacionadas ao excesso de peso ${ }^{11,23,24}$, visto que o aumento na quantidade de gordura corporal está mais freqüentemente associado à alterações metabólicas prejudiciais à saúde $\mathrm{e}^{25,26}$.

O percentual de gordura corporal das mulheres deste estudo foi semelhante ao verificado em outros trabalhos com mulheres de 40 anos ou mais. Por meio do mesmo método (BIA), na po- pulação feminina americana, foram constatadas médias de $\%$ GC de $35,4 \%, 37,3 \%$ e $36,9 \%$, nos grupos etários de 40 a 49 anos, 50 a 59 anos e 60 a 69 anos, respectivamente ${ }^{27}$. Em estudo com mulheres chinesas, foram obtidas médias de 32,6\%, $34,4 \%$ e $35,6 \%{ }^{28}$; e em mulheres inglesas, constataram-se $34,0 \%, 36,8 \%$ e $38,4 \%$ nos mesmos grupos etários $^{29}$.

Enquanto alguns estudos, transversais e longitudinais, indicam associação positiva entre idade e obesidade segundo o IMC $^{29-31}$ e segundo o \%GC em mulheres ${ }^{11,27,29}$, essa relação não foi verificada neste trabalho. Contudo, faz-se necessário ressaltar que a amplitude da idade era maior, nos estudos que mostraram esta relação. 
Tabela 3. Distribuição das mulheres de 40 a 65 anos atendidas em ambulatórios públicos da cidade de São Paulo, segundo obesidade (identificada pelo \%GC) e variáveis sociodemográficas, clínicas e relacionadas ao estilo de vida.

\begin{tabular}{|c|c|c|c|c|c|c|}
\hline \multirow{3}{*}{ Variáveis explanatórias } & \multicolumn{4}{|c|}{ Obesidade (\%GC) } & \multirow{3}{*}{$\mathbf{R P}$} & \multirow{3}{*}{$\begin{array}{c}\text { valor } \\
\text { de } p\end{array}$} \\
\hline & \multicolumn{2}{|c|}{$\begin{array}{c}\text { Não } \\
(\mathbf{n}=353)\end{array}$} & \multicolumn{2}{|c|}{$\begin{array}{c}\text { Sim } \\
(n=116)\end{array}$} & & \\
\hline & $(\mathbf{n})$ & $(\%)$ & $(\mathbf{n})$ & $(\%)$ & & \\
\hline \multicolumn{7}{|l|}{ Fase do climatério } \\
\hline Pré-menopausa $(\mathrm{n}=234)$ & 179 & 76,5 & 55 & 23,5 & 1 & $0,538^{\star}$ \\
\hline Pós-menopausa (n = 235) & 174 & 74,0 & 61 & 26,0 & 1,11 & \\
\hline \multicolumn{7}{|l|}{ Terapia hormonal da menopausa } \\
\hline Nunca usou, já usou ou usa há menos de 12 meses $(\mathrm{n}=415)$ & 308 & 74,2 & 107 & 25,8 & 1 & $0,167^{\star *}$ \\
\hline Usa por, no mínimo, 12 meses $(\mathrm{n}=54)$ & 45 & 83,3 & 9 & 16,7 & 0,65 & \\
\hline \multicolumn{7}{|l|}{ Hábito de fumar } \\
\hline Nunca fumou ou ex-tabagista $(\mathrm{n}=404)$ & 302 & 74,8 & 102 & 25,2 & 1 & 0,528 \\
\hline Tabagista $(\mathrm{n}=65)$ & 51 & 78,5 & 14 & 21,5 & 0,85 & \\
\hline \multicolumn{7}{|l|}{ Nível de atividade física } \\
\hline Sedentária ou insuficientemente ativa $(\mathrm{n}=260)$ & 173 & 66,5 & 87 & 33,5 & 1 & $0,000^{* *}$ \\
\hline Ativa ou muito ativa $(\mathrm{n}=209)$ & 180 & 86,1 & 29 & 13,9 & 0,41 & \\
\hline \multicolumn{7}{|l|}{ Paridade } \\
\hline 0,1 ou 2 partos $(n=120)$ & 101 & 84,2 & 19 & 15,8 & 1 & $0,013^{\star *}$ \\
\hline 3 ou mais partos $(n=349)$ & 252 & 72,2 & 97 & 27,8 & 1,76 & \\
\hline \multicolumn{7}{|l|}{ Anos de estudo } \\
\hline Até 7 anos $(\mathrm{n}=209)$ & 151 & 72,2 & 58 & 27,8 & 1 & $0,174^{* *}$ \\
\hline 8 anos ou mais $(n=260)$ & 202 & 77,7 & 58 & 22,3 & 0,80 & \\
\hline \multicolumn{7}{|l|}{ Idade (variável contínua) } \\
\hline Média & \multicolumn{2}{|c|}{51,2} & \multicolumn{2}{|c|}{51,9} & & 0,317 \\
\hline
\end{tabular}

Apesar da questão do aumento de peso entre mulheres na fase do climatério ser frequentemente relatada, sua relação com a transição da menopausa ainda permanece incompreendida ${ }^{32}$. A diminuição do gasto energético, atribuída às mudanças endócrinas abruptas características do período, fomentou o início de inúmeras investigações sobre a possível relação existente entre a menopausa e o aumento de peso na mulher ${ }^{33,34}$, entretanto, essa relação não foi confirmada por estudos longitudinais posteriores ${ }^{35-37}$, ou foi confirmada por estudos que consideraram o efeito da menopausa pequeno em relação a outros fatores $^{38}$. No presente estudo não foi verificada associação entre a fase do climatério e obesidade, de acordo com os dois critérios adotados (IMC e \%GC).

Deve-se considerar que resultados controversos da literatura sobre a relação da menopausa com as mudanças corporais (aumento do peso, do conteúdo e distribuição da gordura corporal) podem ser decorrentes de diversos fatores, como o uso de diferentes técnicas de mensuração das variáveis antropométricas e critérios de diagnóstico de obesidade, escassez de estudos prospectivos, que possibilitem acompanhamento de mulheres durante a transição menopausal, assim como pela falta de controle por outras variáveis, como a idade e o nível de atividade física.

Neste estudo, a prática insuficiente de atividade física associou-se à maior prevalência de obesidade, segundo o IMC e o \%GC, e este parece ser o fator mais claramente associado ao aumento de peso e da massa gordurosa na mulher, demonstrado amplamente na literatura. Diversos outros estudos também associaram a prática de atividade física regular à prevenção do aumento de peso e das alterações adversas na quantidade e distribuição de gordura corporal em mulheres, frequentemente relacionadas ao envelhecimento e à transição menopausal ${ }^{39-41}$.

Em estudo prospectivo ${ }^{42}$, maior nível de atividade física, menor paridade e maior grau de instrução foram importantes para reduzir o im- 
Tabela 4. Modelo da regressão " $\mathrm{glm}$ " (general linear model), da obesidade (identificada pelo \%GC), em mulheres de 40 a 65 anos, atendidas em ambulatórios públicos da cidade de São Paulo.

\begin{tabular}{|c|c|c|c|c|}
\hline \multirow{2}{*}{$\begin{array}{c}\text { Variáveis } \\
\text { Explanatórias }\end{array}$} & \multicolumn{2}{|l|}{$1^{\text {a }}$ etapa } & \multicolumn{2}{|c|}{ Modelo final } \\
\hline & RP (IC 95\%) & $\begin{array}{l}\text { valor } \\
\text { de p }\end{array}$ & RP (IC 95\%) & $\begin{array}{c}\text { valor } \\
\text { de p }\end{array}$ \\
\hline \multicolumn{5}{|l|}{ Fase do climatério } \\
\hline Pré-menopausa & 1 & & 1 & \\
\hline Pós-menopausa & $1,17(0,86-1,60)$ & 0,314 & $1,15(0,85-1,56)$ & 0,373 \\
\hline \multicolumn{5}{|l|}{ Terapia hormonal da menopausa } \\
\hline Nunca usou, já usou ou usa há menos de 12 meses & 1 & & & \\
\hline Usa por no mínimo 12 meses & $0,68(0,37-1,25)$ & 0,216 & & \\
\hline \multicolumn{5}{|l|}{ Nível de atividade física } \\
\hline Sedentária ou insuficientemente ativa & 1 & & 1 & \\
\hline Ativa ou muito ativa & $0,43(0,30-0,63)$ & 0,000 & $0,43(0,29-0,63)$ & 0,000 \\
\hline \multicolumn{5}{|l|}{ Paridade } \\
\hline 0,1 ou 2 partos & 1 & & 1 & \\
\hline 3 ou mais partos & $1,54(0,99-2,38)$ & 0,056 & $1,60(1,03-2,49)$ & 0,035 \\
\hline \multicolumn{5}{|l|}{ Anos de estudo } \\
\hline Até 7 anos & 1 & & & \\
\hline 8 anos ou mais & $0,81(0,60-1,10)$ & 0,176 & & \\
\hline
\end{tabular}

pacto do aumento do IMC em mulheres na pré e na pós-menopausa. Estudo populacional norte -americano confirmou a contribuição da prática de atividade física regular para prevenir ou atenuar o aumento de peso e na circunferência da cintura em mulheres de 42 a 52 anos, em 3 anos de seguimento ${ }^{12}$.

Em 2014, 56,0\% das mulheres adultas brasileiras foram consideradas insuficientemente ativas, percentual que aumentou com a elevação da idade, e a diminuir com o aumento da escolaridade $^{21}$. A proporção de mulheres brasileiras insuficientemente ativas foi semelhante à observada no presente estudo (55,4\%), porém, cabe ressaltar que foram utilizados critérios diferentes para classificação da prática de atividade física.

Neste estudo, o nível de instrução associouse negativamente à obesidade identificada pelo IMC. Dados sobre a prevalência de obesidade segundo quartis de escolaridade em 37 países em desenvolvimento mostraram que a probabilidade de obesidade nos países com menor nível socioeconômico excedeu a mesma probabilidade naqueles com maior nível socioeconômico ${ }^{43}$. Inquéritos populacionais brasileiros confirmam que a tendência de obesidade reflete o aumento das desigualdades, com o deslocamento do maior risco para adultos de menores estratos de renda e educação ${ }^{13}$.

Dados do Ministério da Saúde do Brasil ${ }^{10}$ mostraram a tendência de diminuição da fre- quência de obesidade, medida pelo IMC, com o aumento do nível de escolaridade, sendo essa relação uniforme entre as mulheres de 27 estados brasileiros: a prevalência de obesidade foi de $24,8 \%$ em mulheres com 0 a 8 anos de estudo, $17,2 \%$ naquelas com 9 a 11 anos de estudo e 10,6\% naquelas com 12 anos ou mais de estudo.

Em outros países com cenário socioeconômico distinto, esta relação também foi estudada. $\mathrm{Na}$ China, a prevalência de obesidade é menor do que nos países ocidentais, porém, estudo representativo com indivíduos de 18 a 74 anos, também constatou a relação inversa do risco de obesidade, segundo o IMC, ao nível educacional, em homens e mulheres ${ }^{44}$. Na Finlândia, o nível de escolaridade também esteve inversamente associado ao IMC em mulheres de 30 a $64 \operatorname{anos}^{45}$. De forma semelhante aos achados do presente estudo, foi encontrada associação inversa entre grau de instrução e IMC, enquanto a mesma relação não foi encontrada com o \%GC, em mulheres iranianas de 18 a 40 anos $^{46}$.

Com relação à história reprodutiva, enquanto sua associação ao excesso de peso na população feminina vem sendo amplamente demonstrada na literatura internacional, no Brasil poucos estudos tiveram como objetivo verificar esta relação. Mais recentemente, no sul do Brasil, a menarca precoce (variável não verificada no presente estudo) e a paridade foram os mais fortes preditores da obesidade em mulheres de 40 a 65 
anos residentes em Caxias do Sul, após ajuste por variáveis demográficas, socioeconômicas e comportamentais $^{47}$. Em 465 mulheres de 45 a 69 anos, residentes na área urbana de Maringá, os fatores que mais fortemente associaram-se ao excesso de peso foram: ter três ou mais partos (OR: 1.78; 95\% IC: 1.06-3.00) e não usar THM (OR: 1.69; 95\% IC: $1.06-2.63)^{48}$.

A análise dos dados da Pesquisa Nacional de Demografia e Saúde (PNDS), provenientes de inquérito realizado em todas as regiões do território brasileiro no ano de 2006, mostraram que a paridade exerce influência sobre a obesidade nas mulheres brasileiras em idade reprodutiva, sendo a associação do tipo dose-resposta, ou seja, mais pronunciada entre as mulheres que possuem filhos do que entre as nulíparas ${ }^{14}$.

No presente estudo, mesmo após controle pelo nível de atividade física, maior paridade manteve-se associada à obesidade de acordo com o \%GC, embora outros estudos não confirmem essa associação. O \%GC, obtido a partir de métodos radiológicos como a DEXA e a tomografia computadorizada, apresentou-se associado à paridade em mulheres de 18 a 76 anos porém, após ajuste por idade, hábito de fumar e nível de atividade física, esta relação não permaneceu significativa ${ }^{49}$.

Com relação ao hábito de fumar, embora alguns estudos mostrem valores médios de IMC menores em indivíduos tabagistas, comparados aos não tabagistas e, também, que poderia haver tendência de aumento de peso naqueles que abandonam o tabagismo ${ }^{44,50,51}$, não foi constatada associação entre hábito de fumar e obesidade neste estudo, da mesma forma que o observado com a terapia hormonal da menopausa (THM).
Não há evidências de que a reposição estrogênica propicie aumento de peso em mulheres na pós-menopausa ${ }^{37,48,52}$ ou, então, que a THM poderia prevenir o aumento de peso em mulheres nessa fase da vida ${ }^{53,54}$. Apesar dos dados sugestivos de estudos realizados com animais, a literatura atual não fornece evidências convincentes de que a terapia estrogênica atenuaria o aumento de peso em mulheres na pós menopausa ${ }^{55}$.

Apesar da dificuldade para comparação dos resultados, devido a utilização de diferentes métodos e critérios para o diagnóstico da obesidade, associações bastante conclusivas entre paridade e nível de atividade física ao desfecho obesidade foram demonstrados em diversos estudos com mulheres, além do presente.

Não obstante, este estudo apresenta certas limitações para extrapolação dos seus resultados, relacionadas principalmente ao delineamento transversal e à amostragem por conveniência. Estudos de abrangência nacional são necessários para investigar a prevalência de obesidade e fatores associados na população feminina brasileira, de modo a oferecer subsídios para a formulação de políticas públicas voltadas à saúde integral da mulher.

\section{Conclusão}

Constatou-se obesidade, segundo o IMC, em $32,0 \%$ das mulheres e, segundo o \%GC, em $24,7 \%$ delas. A prevalência foi maior nas sedentárias, insuficientemente ativas e naquelas que referiram $\geq 3$ partos. Neste estudo, a atividade física constitui-se como fator de proteção, enquanto a paridade ( $\geq 3$ partos) representou fator de risco para a obesidade.

\section{Colaboradores}

AP França trabalhou na concepção e delineamento do projeto, coleta, análise, interpretação e discussão dos dados e da redação do artigo; MFN Marucci trabalhou na concepção e delineamento do projeto, análise, interpretação e discussão dos dados e revisão crítica do artigo; MLN Silva e MA Roediger participaram da interpretação e discussão dos dados e revisão crítica do artigo.

\section{Agradecimentos}

À Coordenação de Aperfeiçoamento de Pessoal de Nível Superior (Capes) pela concessão da bolsa de estudos. 


\section{Referências}

1. World Health Organization (WHO). Global recommendations on physical activity for health. Geneva: WHO; 2010.

2. Malik VS, Willett WC, Hu FB. Global obesity: trends, risk factors and policy implications. Nat Rev Endocrinol 2013; 9(1):13-27.

3. Finucane MM, Stevens GA, Cowan MJ, Danaei G, Lin JK, Paciorek CJ, Singh GM, Gutierrez HR, Lu Y, Bahalim AN, Farzadfar F, Riley LM, Ezzati M; Global Burden of Metabolic Risk Factors of Chronic Diseases Collaborating Group (Body Mass Index). Global Burden of Metabolic Risk Factors of Chronic Diseases Collaborating Group (Body Mass Index). National, regional, and global trends in body-mass index since 1980: systematic analysis of health examination surveys and epidemiological studies with 960 country-years and 9.1 million participants. Lancet 2011; 377(9765):557-567.

4. World Health Organization (WHO). World health statistics 2015. Geneva: WHO; 2015.

5. Prospective Studies Collaboration. Body-mass index and cause-specific mortality in 900000 adults: collaborative analyses of 57 prospective studies. Lancet 2009; 373(9669):1083-1096

6. World Health Organization (WHO). Global health risks: mortality and burden of disease attributable to selected major risks. Geneva: WHO; 2009.

7. World Health Organization (WHO). Global status report on noncommunicable diseases 2010. Geneva: WHO 2011.

8. Malta DC, Moura L, Prado RR, Escalante JC, Schmidt MI, Duncan BB. Mortalidade por doenças crônicas não transmissíveis no Brasil e suas regiões, 2000 a 2011. Epidemiol Serv Saúde 2014; 23(4):599-608.

9. Brasil. Ministério da Saúde (MS). Vigitel Brasil 2006: vigilância de fatores de risco e proteção para doenças crônicas por inquérito telefônico. Brasília: MS; 2007.

10. Brasil. Ministério da Saúde (MS). Vigitel Brasil 2012: vigilância de fatores de risco e proteção para doenças crônicas por inquérito telefônico. Brasília: MS; 2013.

11. Gallagher D, Heymsfield SB, Heo M, Jebb AS, Murgatroyd PR, Sakamoto Y. Healthy percentage body fat ranges: an approach for developing guidelines based on body mass index. Am J Clin Nutr 2000; 72(3):694-701.

12. Sternfeld B, Wang H, Quesenberry CP Jr, Abrams B, Everson-Rose SA, Greendale GA, Matthews KA, Torrens JI, Sowers M. Physical activity and changes in weight and waist circumference in midlife women: findings from the Study of Women's Health Across the Nation. Am J Epidemiol 2004; 160(9):912-922.

13. Monteiro CA, Conde WL, Popkin BM. Income-specific trends in obesity in Brazil: 1975-2003. Am J Public Health 2007; 97(10):1808-1812.

14. Ferreira RAB, Benicio MHDA. Obesidade em mulheres brasileiras: associação com paridade e nível socioeconômico. Rev Panam Salud Publica 2015; 37(4/5):337342.

15. World Health Organization (WHO). Obesity: preventing and managing the global epidemic. Geneva: WHO; 1997. (Report of a WHO consultation on obesity).

16. Matsudo S, Araújo T, Matsudo V, Andrade D, Andrade E, Oliveira LC, Braggion G. Questionário Internacional de Atividade Física (IPAQ): estudo de validade e reprodutibilidade no Brasil. Rev Bras Ativ Fís Saúde 2001; 6(2):5-18.
17. Benedetti TB, Mazo GZ, Barros MVG. Aplicação do questionário internacional de atividades físicas para avaliação do nível de atividades físicas de mulheres idosas: validade concorrente e reprodutibilidade teste -reteste. Rev Bras Ciên e Mov 2004; 12(1):25-33.

18. Monteiro CA. Epidemiologia da obesidade. In: Halpern A, organizador. Obesidade. São Paulo: Editora Lemos; 1998. p. 15-30.

19. Instituto Brasileiro de Geografia e Estatística (IBGE). Pesquisa de Orçamentos Familiares 2002-2003. Rio de Janeiro: IBGE; 2004.

20. Instituto Brasileiro de Geografia e Estatística (IBGE). Pesquisa de Orçamentos Familiares 2008-2009: antropometria e estado nutricional de crianças, adolescentes e adultos no Brasil. Rio de Janeiro: IBGE; 2010.

21. Brasil. Ministério da Saúde (MS). Vigitel Brasil 2014: vigilância de fatores de risco e proteção para doenças crônicas por inquérito telefônico. Brasília: MS; 2015.

22. Ogden CL, Carroll MD, Kit BK, Flegal KM. Prevalence of obesity among adults: United States, 2011-2012. NCHS data brief, no 131. Hyattsville: National Center for Health Statistics; 2013.

23. Frankenfield DC, Roew WA, Cooney RN, Smith JS, Becker D. Limits of body mass index to detect obesity and predict body composition. Nutrition 2001; 17(1):26-30.

24. Zhu S, Wang Z, Shen W, Heymsfield SB, Heshka S. Percentage body fat ranges associated with metabolic syndrome risk: results based on the third National Health and Nutrition Examination Survey (1988-1994). Am J Clin Nutr 2003; 78(2):228-235.

25. Shea JL, King MTC, Yi Y, Gulliver W, Sun G. Body fat percentage is associated with cardiometabolic dysregulation in BMI-defined normal weight subjects. Nutr Metab Cardiovasc Dis 2012; 22(9):741-747.

26. Bastien M, Poirier P, Lemieux I, Després J-P. Overview of Epidemiology and Contribution of Obesity to Cardiovascular Disease, Prog Cardiovasc Dis 2014; 56(4):369-381.

27. Chumlea WC, Guo SS, Kuczmarski RJ, Flegal KM, Johnson CL, Heymsfield SB, Lukaski HC, Friedl K, Hubbard VS. Body composition estimates from NHANES III bioelectrical impedance data. Int J Obes 2002; 26(12):1596-609.

28. Wang C, Hou X, Zhang M, Bao Y, Zou Y, Zhong W, Xiang KS, Jia WP. Comparison of Body Mass Index with Body Fat Percentage in the Evaluation of Obesity in Chinese. Biomed Environ Sci 2010; 23(3):173-179.

29. Meeuwsen S, Horgan GW, Elia M. The relationship between BMI and percent body fat, measured by bioelectrical impedance, in a large adult sample is curvilinear and influenced by age and sex. Clin Nutr 2010; 29(5):560-566.

30. Hjartaker A, Adami H-O, Lund E, Weiderpass E. Body mass index and mortality in a prospectively studied cohort of Scandinavian women: the women's lifestyle and health cohort study. Eur J Epidemiol 2005; 20:747-754.

31. Hardy R, Mishra GD, Kuh D. Body mass index trajectories and age at menopause in a British birth cohort. Maturitas 2008:59(4):304-314.

32. Wildman RP, Sowers MR. Adiposity and the Menopausal Transition. Obstet Gynecol Clin North Am 2001; 38(3):441-454. 
33. Poehlman ET, Goran MI, Gardner AW, Ades PA, Arciero PJ, Katzman-Rooks SM, Montgomery SM, Toth MJ, Sutherland PT. Determinants of decline in resting metabolic rate in aging females. Am J Physiol 1993; 264(3 Pt 1):E450-455.

34. Poehlman ET, Tchernof A. Transversing the menopause: changes in energy expenditure and body composition. Coron Artery Dis 1998; 9(12):799-803.

35. Crawford SL, Casey VA, Avis NE, McKinlay SM. A Longitudinal Study of Weight and the Menopause Transition: Results From the Massachusetts Women's Health Study. Menopause 2000; 7(2):96-104.

36. Blümel JE, Castelo-Branco C, Rocangliolo ME, Bifa L, Tacla X, Mamani L. Changes in body mass index around menopause: a population study of Chilean woman. Menopause 2001; 8(4):239-244.

37. Macdonald HM, New SA, Campbell MK, Reid DM. Longitudinal changes in weight in perimenopausal and early postmenopausal women: effects of dietary energy intake, energy expenditure, dietary calcium intake and hormone replacement therapy. Int J Obes 2003; 27(6):669-676.

38. Matthews KA, Abrams B, Crawford S, Miles T, Neer $\mathrm{R}$, Powell LH, Wesley D. Body mass index in mid-life women: relative influence of menopause, hormone use, and ethnicity. Int J Obes 2001; 25(6):863-873.

39. Gilliat-Wimberly M, Manore MM, Woolf K, Swan PD, Carroll SS. Effects of habitual physical activity on the resting metabolic rates and body compositions of women aged 35 to 50 years. J Am Diet Assoc 2001; 101(10):1181-1188.

40. Sternfeld B, Bhat AK, Wang H, Sharp T, Quesenberry Junior CP. Menopause, physical activity, and body composition/fat distribution in midlife women. Med Sci Sports Exerc 2005; 37(7):1195-1202.

41. Arsenault BJ, Rana JS, Lemieux I, Després JP, Kastelein JJ, Boekholdt SM, Wareham NJ, Khaw KT. Physical inactivity, abdominal obesity and risk of coronary heart disease in apparently healthy men and women. Int $J$ Obes (Lond) 2010; 34(2):340-347.

42. Hjartaker A, Adami H-O, Lund E, Weiderpass E. Body mass index and mortality in a prospectively studied cohort of Scandinavian women: the women's lifestyle and health cohort study. Eur J Epidemiol 2005; 20(9):747754.

43. Monteiro CA, Moura EC, Conde WL, Popkin BM. Socioeconomic status and obesity in adult populations of developing countries: a review. Bull World Health Organ 2004; 82(12):940-946.

44. Wang H, Wang J, Liu MM, Wang D, Liu Y-Q, Zhao Y, Huang MM, Liu Y, Sun J, Dong GH. Epidemiology of general obesity, abdominal obesity and related risk factors in urban adults from 33 communities of northeast china: the CHPSNE study. BMC Public Health 2012; 12:967.

45. Seppänen-Nuijten E, Lahti-Koski M Männistö S, Knekt, P, Rissanen H, Aromaa A, Heliövaara M. Fat free mass and obesity in relation to educational level. BMC Public Health 2009; 9:448.
46. Amani R. Comparison between bioelectrical impedance analysis and body mass index methods in determination of obesity prevalence in Ahvazi women. Int $J$ Obes 2007; 61:478-82.

47. Theodoro H, Rodrigues AD, Mendes KG, Liane RH, Paniz VMV, Olinto MTA. Reproductive characteristics and obesity in middle-aged women seen at an outpatient clinic in southern Brazil. Menopause 2012; 19(9):1022-1028.

48. Gravena AA, Brischiliari SC, Lopes TC, Agnolo CM, Carvalho MD, Pelloso SM. Excess weight and abdominal obesity in postmenopausal Brazilian women: a population-based study. BMC Women's Health 2013; 13:46.

49. Blaudeau TE, Hunter GR, Sirikul B. Intra-abdominal adipose tissue deposition and parity. Int J Obes 2006; 30(7):1119-1124.

50. Froom P, Melamed S, Benbassat J. Smoking cessation and weight gain. Fam Pract 1998; 46(6):460-464.

51. Owen-Smith V, Hannaford PC. Stopping smoking and body weight in women living in the United Kingdom. Br J Gen Pract 1999; 49(449):989-990.

52. Kongnyuy EJ, Norman RJ, Flight IHK, Rees MC. Oestrogen and progestogen hormone replacement therapy for peri-menopausal and post-menopausal women: weight and body fat distribution. Cochrane Database Syst Rev 2000; (2):CD001018.

53. Gambacciani M, Ciaponi M, Cappagli B. De Simone L, Orlandi R, Genazzani AR. Prospective evaluation of body weight and body fat distribution in early postmenopausal women with and without hormonal replacement therapy. Maturitas 2001; 39:125-132.

54. Genazzani AR, Gambacciani M. Effect of climacteric transition and hormone replacement therapy on body weight and body fat distribution. Gynecol Endocrinol 2006; 22(3):145-150.

55. Springer AM, Foster-Schubert K, Morton GJ, Schur EA. Is there evidence that estrogen therapy promotes weight maintenance via effects on leptin? Menopause 2014; 21(4):424-432.

Artigo apresentado em 30/11/2015

Aprovado em 13/12/2016

Versão final apresentada em 15/12/2016 